\title{
Subjectivités senghoriennes : pour une prospective francophone
}

\author{
Mbaye Diouf \\ Université McGill
}

\section{Résumé}

Comment re-lire Senghor à l'ère de la pandémie du coronavirus ? Cet article se veut un pari de prospective dans un présent et un futur immédiat qui semblent graduellement pris au piège d'une terminologie négative : épidémies, terrorismes, radicalismes, replis, rejets, exils, «identités meurtrières », etc. Senghor aiderait certainement à sortir de ce piège terminologique par sa formulation poétique et discursive d'une « altérité dynamique », seule capable de relier un jeune poète chrétien noir des années 30 à l'anomie pandémique des années 2020. Mais sans doute aussi seule capable de porter un catholique pratiquant sérère à la présidence d'un pays africain très majoritairement musulman. L'objectif ici est de relier l'œuvre du poète sous l'angle des «subjectivités senghoriennes », subjectivités perçues non pas comme des chimères lointaines mais comme un exorcisme des dangers contemporains par le biais d'une philosophie pratique du dialogue interreligieux, une éthique politique du vivreensemble et une poésie de l'empathie qui résoudraient bien des ruptures de notre temps.

Mots-clés : Senghor, poésie, énonciation, habilité, agentivité 
L'on ne peut être plus subjectif que Senghor, penserait-on... si l'on se réfère à la francité de l'ancien Président de la République du Sénégal, à la négritude de l'Académicien noir ou à la catholicité du poète sérère. On peut, en effet, être porté à croire que Léopold Sédar Senghor est l'incarnation même des assemblages inédits, l'artificier des unions surprenantes voire contradictoires, le penseur des possibles impensables. Bref, l'auteur africain qui, de loin, aura résolument porté une subjectivité du monde à la fois comme matière poétique, comme projet politique et comme objet épistémologique. Cela nous ramène à l'étymologie même du mot « subjectivité », c'est-à-dire une certaine relation au monde, l'émergence d'un « Sujet » au centre de «l'enjeu d'être face à autrui » (Homi Bhabha, 2007 : 16), mais aussi à un ensemble de dispositions et de postures propres à ce même Sujet (ses abilities) qui déterminent sa conviction et son action sur un monde réformable (son agency). La «subjectivité » est donc affaire d'énonciation, comme l'indiquent Kerbrat-Orecchioni (2005) et Amidou Sanogo (2021), et chez Senghor, c'est une affaire d'énonciation spécifique d'un croire et d'un faire dans un contexte historique déterminé. Une énonciation spécifique clairement portée par un «Je » proéminent qui circule dans toute l'œuvre du poète, tous genres confondus :

Seigneur, éloigne de ma mémoire la France qui n'est pas la France [...] ce masque de petitesse et de haine pour qui je n'ai que haine. Vous, Tirailleurs sénégalais, [...] Je ne laisserai pas la parole aux ministres, et pas aux généraux. Je ne laisserai pas - non! - les louanges de mépris vous enterrer furtivement.

Je déchirerai les rires banania sur tous les murs de France.

Je confesserai même - Aragon m'en donne l'exemple - que j'ai beaucoup lu, des troubadours à Paul Claudel. 
La vérité est que j'ai surtout lu, plus exactement écouté, transcrit et commenté des poèmes négro-africains.

Ma Négritude est truelle à la main. (1948 : 67-96) [je souligne]

Ce « Je » de l'intention, de l'action et de la projection est le noyau matriciel des abilities et des agencies senghoriens. Il est le signe linguistique fondateur qui signale une érudition, donne corps à une intime conviction et détermine les paramètres d'un langage poétique et réflexif particulier. Et Senghor n'a cessé de le rappeler : «Toute ma vie, j'ai cru en certains principes et idées. C'est que je les avais sentis comme des vérités enracinées dans les faits, dans la vie, et dès les premières années de mon existence sur la terre des hommes » (1988:9). Il s’agira alors d'analyser ici les mécanismes d'énonciation d'une subjectivité senghorienne «enracinée dans les faits » et destinée à réformer le monde en mobilisant toutes les abilities vers une performance des agencies.

\section{« Le siècle de Senghor » (1906-2001)}

Le $\mathrm{XX}^{\mathrm{e}}$ siècle est un siècle riche en événements majeurs qui font de Senghor un témoin privilégié des tournants décisifs du monde contemporain. Il existe, en effet, une forme d'historicité dynamique qui particularise ce siècle et relie entre eux tous ces tournants décisifs, de sorte qu'il est peut-être plus approprié de parler d'une accumulation de « temps » qui unit les principaux événements du siècle en plusieurs temps forts substantiellement indissociables. J'en mentionnerai quatre : - un temps des transitions historiques qui relie de manière polémique des formes d'administration et de domination coloniales à des formes d'émancipation et d'expression de type postcolonial (Yves Clavaron, 2017). 
- un temps des dénis humains qui atteint son paroxysme dans des formes de négation humaine comme la Shoah ou le génocide des Tutsis du Rwanda (Josias Semujanga, 2008).

- un temps des cohabitations compliquées qui porte à redéfinir les notions fondatrices d'État et de Nation et leurs antagonismes contemporains dans des formules uniculturelles, interculturelles ou multiculturelles (Charles Taylor, 2009).

- enfin un temps des radicalismes sacralisés qui trouvent leurs ultimes manifestations dans un «extrême contemporain » (Michel Chaillou, 1986) marqué par un retour brutal des fondamentalismes religieux et des replis communautaires.

Senghor est contemporain de ces quatre « temps » qu'il n'aborde pas de manière séparée dans ses œuvres, mais plutôt de manière convergente dans un cinquième temps énonciatif que je dénommerai ici un «temps senghorien de la $5^{\mathrm{e}}$ voie ».

Ce «temps senghorien de la $5^{\mathrm{e}}$ voie » est en quelque sorte un vaste projet de relecture personnelle de l'histoire de l'Humanité pour, d'une part, réinventer une nouvelle « Civilisation de l'Universel » destinée à amortir et à prévenir les chocs ravageurs des quatre temps cités plus haut, et, d'autre part, à penser l'espace francophone comme un croisement de faits socioculturels dynamiques qui ajuste continuellement les relations historiques entre les peuples et les États de cet espace avec la France. Ce croisement nourrit en même temps les temporalités sociopolitiques de ces peuples et de ces États, articule leurs économies formelles et informelles, influence leur vécu linguistique et idiomatique, et inspire leurs créations artistiques et artisanales. 
Le «temps senghorien de la $5^{\mathrm{e}}$ voie » est donc un temps de redéfinition des négociations internationales, un temps de valorisation des interrelations humaines et un temps d'implication communautaire, comme le montre si bien le devenir global du monde à l'ère du Coronavirus. Car le salut de l'humanité n'est pas dans la promotion d'une civilisation occidentale hégémonique qui s'octroie souvent militairement un incommensurable droit d'universalisme, mais dans une «Civilisation de l'Universel» à bâtir et à rebâtir continuellement et communément «par tous les peuples de la terre » $(1988: 204)$ et dans laquelle les notions de hiérarchie, de possession et de domination feront place à celles de relativité, de partage et d'équité. Le salut de l'humanité n'est pas non plus dans la mutation définitive de l'outil technologique en moyen ultime de domination d'un peuple sur l'autre, mais dans le devoir de résister « au joug de la matière » sur la «primauté de la culture » $(1988: 211)$. Le salut de l'humanité n'est pas enfin dans la célébration d'un modèle social unique, mais dans la reconstruction des sociétés humaines modernes autour des valeurs de diversité, d'échanges et de pluralité qui sont les conditions même d'expression et de réussite du fait francophone dynamique.

Comme l'indique Senghor dans son Discours de réception à l'Académie française, c'est à l'aune de ce fait francophone objectif, quotidien et multiforme qu'il faut lire et analyser le rapport de l'Afrique au Monde à partir d'une heuristique systématique et ouverte. L'heuristique, au sens du poète,

consiste à découvrir les faits significatifs d'une histoire ou d'un phénomène $[\ldots]$ Le fait ainsi découvert, il faut rechercher tous ses tenants et ses aboutissants : ses causes, ses effets, sa signification, sa valeur pour les acteurs et les contemporains... pour nous. (1984: 14) 
La clé de l'intérêt particulier de Senghor pour l'Histoire générale tient précisément à cette perception de l'heuristique comme principe de connaissance du Même, de l'Autre et du Monde :

Je ne savais pas encore que mon nom venait du portugais, avec quelques gouttes de sang européen. Si je l'avais su, j'en aurais non pas rougi mais sué. Les griots, nos troubadours, m'avaient seulement appris que les ancêtres de mon père appartenaient au grand peuple mandingue dont les Malinkés, l'ethnie la plus guerrière et paradoxalement la plus artiste, avaient, en venant du Sud, conquis le royaume du Sine il y a plusieurs siècles. Cependant, se comportant en guerriers pacifiques, ils avaient épousé des filles de la noblesse, se faisant Sérères avec les Sérères. (1988: 11)

Découvrir le fait d'origine, en suivre la trace et ses ramifications, en déduire les configurations présentes et formuler les possibles à venir sont là quelques principes heuristiques de la pensée et de la poésie de Senghor tout au long du $\mathrm{XX}^{\mathrm{e}}$ siècle. Intuition précoce, peut-on dire, car étant au centre de ses intérêts scolaires dès le début, de la Mission Catholique de Ngazobil, petite bourgade près de Joal où il effectue son cycle primaire, au Collège-Séminaire Libermann de Dakar, lieu de la scolarité secondaire et des rêves avortés de prêtrise, puis au Lycée Van Vollenhoven de Dakar, lieu laïc de la maturation scolaire avant l'arrivée en France à la fin des années 20. Outre son mandat de député du Sénégal au Parlement français dans les années 40 et 50, période pendant laquelle il enseigne aussi les langues et les civilisations négro-africaines à l'École nationale de la France d'outre-Mer (ENFOM), la carrière professionnelle de Senghor en France confirme cette intuition précoce qu'une présence assumée au monde passe d'abord par une connaissance tangible des faits constitutifs du Soi, de la communauté d'origine et des relations internationales. 
Le « temps senghorien de la $5^{\mathrm{e}}$ voie » émane et relie substantiellement toutes les périodes de cette trajectoire personnelle pour laisser voir non pas un ensemble hétéroclite d'une vie éclatée entre le Sine et la Seine, mais plutôt la lente maturation poétique et philosophique d'une heuristique du fait francophone dans lequel Senghor se veut à la fois le produit (l'héritier des humanités africaine et française) et l'agent (l'acteur d'une institution francophone multiculturelle et inclusive). Des recueils de poésies (Euvre poétique) aux livres de jeunesse ( $\mathrm{La}$ belle histoire de Leuk-le-lièvre), des anthologies (Anthologie de la nouvelle poésie nègre et malgache de langue française) aux essais (Liberté I à Liberté V), tous les écrits senghoriens participent de ce vaste projet dans lequel «être francophone » est à la fois un fait identitaire et une position de discours.

Senghor compte, en effet, inscrire sa pensée réformatrice dans une histoire globale de la pensée du monde et de l'humain. Il ne s'agit nullement de s'isoler dans une Négritude de type raciste voire épidermique qui supprime toute forme d'altérité ou « emprisonne le Noir dans sa noirceur », mais plutôt de réclamer une Négritude humaniste et participative qui est « une certaine manière d'être homme, surtout de vivre en homme» (1988: 139), mais également un moyen de «s'ouvrir ensuite aux apports fécondants des autres civilisations» (1989: 161). Or, «être homme» est une équation épistémologique mille fois posée par l'historiographie européenne, continuellement mise en scène par la grande littérature occidentale, régulièrement glorifiée par la geste des Nations coloniales. Charlemagne, Louis XIV, Napoléon, tout comme Ulysse, Macbeth ou Don Quichotte personnifient cette mystique occidentale de la force, de la grandeur, du leadership, de la conquête, de l'ingéniosité, de l'empathie, du progrès ou de l'intelligence. Ces «héros occidentaux» ont pu entretenir des héritages politiques, configurer des valeurs de référence, alimenter une philosophie de 
l'hégémonisme, instaurer des icônes littéraires et modéliser une certaine façon occidentale d'être au monde.

Dans la formulation du «temps senghorien de la $5^{\mathrm{e}}$ voie », cette « certaine façon occidentale d'être au monde » ne saurait être l'unique façon d'être au monde. Il faut donc interroger ses mythes fondateurs, remonter à sa prime inspiration, en déconstruire l'idée conductrice et la mission civilisatrice. C'est la raison pour laquelle dans la formation de l'CEuvre senghorienne, l'historien Senghor devance le poète Senghor, le grammairien Senghor devance l'écrivain Senghor, le linguiste Senghor devance le mécène culturel Senghor. Pour dire l'Afrique, et à travers elle le fait francophone dans toute sa diversité artistique, socioéconomique et culturelle, Senghor procède à une critique systématique de l'histoire des pensées et des concepts qui ont longtemps encadré, illustré et défini le monde à partir d'une centralité européenne. Le Discours de réception à l'Académie française est un exemple d'intertextualité citative qui étudie minutieusement les plus grands historiens de l'histoire de France, d'Antoine de Lévis-Mirepoix à Jules Michelet, en passant par Paul Rivet et André Malraux. Perspective intertextuelle reprise et élargie dans ce que l'on peut désormais considérer comme le livre-testament de Senghor, Ce que je crois. Négritude, Francité et Civilisation de l'Universel (1988).

Le but, dans ces ouvrages, n'est pas de faire un simple bilan diachronique de l'histoire de la France et de l'Europe, mais de procéder à une heuristique des faits historiques et des principes idéologiques qui ont façonné le rapport colonial des puissances européennes et singulièrement de la France avec l'Afrique et les autres territoires francophones. Ce rapport colonial s'est d'abord justifié de la certitude d'une altérité verticale qui hiérarchise les cultures et les sociétés, qui 
ordonne de manière pyramidale les races, classe les peuples par degrés d'évolution et finit par décider du «droit» de certains peuples sur d'autres, comme l'avoue officiellement le député Jules Ferry à la séance parlementaire du 28 juillet 1885 :

Voilà, messieurs, la thèse [...] Je disais, pour appuyer cette proposition, à savoir qu'en fait, comme on le dit, la politique d'expansion coloniale est un système politique et économique, je disais qu'on pouvait rattacher ce système à trois ordres d'idées : à des idées économiques, à des idées de civilisation de la plus haute portée et à des idées d'ordre politique et patriotique [...]

Messieurs, il faut parler plus haut et plus vrai ! Il faut dire ouvertement qu'en effet les races supérieures ont un droit vis-à-vis des races inférieures... Je répète qu'il y a pour les races supérieures un droit, parce qu'il y a un devoir pour elles. Elles ont le devoir de civiliser les races inférieures... $(1885)^{35}$

Père de l'instruction publique française, ancien ministre et député républicain de Paris longtemps opposé à l'Empire, Jules Ferry n'est pas un colonialiste circonstanciel ni un de ces nombreux parlementaires quasi anonymes de l'époque. Orateur reconnu du personnel politique français de la III ${ }^{\mathrm{e}}$ République, il est l'un des idéologues les plus audibles de «la politique d'expansion coloniale». Position paradoxale, pourrait-on penser, car dans la planification comme dans l'exécution, cette politique est d'abord l'œuvre de la Monarchie et de l'Empire

35 Jules Ferry ajoute plus loin : «Est-ce que vous pouvez nier, est-ce que quelqu'un peut nier qu'il y a plus de justice, plus d'ordre matériel et moral, plus d'équité, plus de vertus sociales dans l'Afrique du Nord depuis que la France a fait sa conquête ? Quand nous sommes allés à Alger pour détruire la piraterie et assurer la liberté du commerce dans la Méditerranée, est-ce que nous faisions œuvre de forbans, de conquérants, de dévastateurs ? Est-il possible de nier que, dans l'Inde, et malgré les épisodes douloureux qui se rencontrent dans l'histoire de cette conquête, il y a aujourd'hui infiniment plus de justice, plus de lumière, d'ordre, de vertus publiques et privées depuis la conquête anglaise qu'auparavant ? [...] Est-ce qu'il est possible de nier que ce soit une bonne fortune pour ces malheureuses populations de l'Afrique équatoriale de tomber sous le protectorat de la nation française ou de la nation anglaise ?» (1885, en ligne). 
français du XIX ${ }^{\mathrm{e}}$ siècle, systèmes politiques pourtant frontalement combattus par Jules Ferry au profit d'une « République vertueuse et égalitaire » qui incarnerait « la civilisation française de la plus haute portée » (1885). C'est que Jules Ferry est un héritier convaincu d'une épopée européenne de la supériorité et de la centralité largement propagée par la littérature (Voltaire, Victor Hugo, Diderot), la philosophie (Montesquieu, Hegel, David Hume, Renan) et l'Église (Pape Nicolas V, Pape Léon XIII). La défense de « la politique d'expansion coloniale » est une logique objective de l'histoire, un « devoir » plus qu'un « droit », préciset-il dans son discours.

Député du Sénégal un demi-siècle plus tard dans cette même Chambre des Représentants, Senghor mesurera la nécessité de poser autrement la question du fait francophone issu de la relation coloniale avec la France. Non plus déterminé par une altérité verticale qui transforme le projet colonial en mission civilisatrice européenne, ce fait francophone doit plutôt se refonder sur une interrelation horizontale qui reconnait une civilisation en tout peuple et une égale dignité de l'ensemble des civilisations humaines. Et je réitère ici, après l'avoir démontré ailleurs (2016), que le principal lieu d'ancrage de la poésie et de l'essai senghoriens n'est pas la célébration lyrique du « royaume d'enfance » mais bien une révision heuristique d'une supposée hiérarchie des civilisations humaines établie à partir de catégories occidentales. Le Senghor historien a de ce fait fondamentalement devancé et inspiré le Senghor poète. C'est précisément ce Senghor historien qui est en quête d'une $5^{\mathrm{e}}$ voie à même de désamorcer les cristallisations dévastatrices du $\mathrm{XX}^{\mathrm{e}}$ siècle et qui n'hésite pas à rappeler l'antériorité des civilisations africaines : 
Les Africains sont restés «aux avant-postes de la Civilisation» jusqu'au Paléolithique supérieur, il y a 40000 ans. Et moi, je dis avec Paul Rivet, l'un des fondateurs de l'anthropologie moderne: jusqu'au IV $^{\mathrm{e}}$ millénaire avant notre ère, quand les Égyptiens inventèrent la première écriture. Je sais que certains leur ont contesté cette priorité pour l'attribuer aux Sumériens. Je précise que même si cette dernière thèse était retenue, le problème ne changerait pas au fond : les Sumériens étaient un peuple de couleur parlant une langue agglutinante, tout comme les Noirs dravidiens de l'Inde qui, vers l'an 2500 avant notre ère, inventèrent la troisième grande écriture $[. .$.$] En$ vérité, c'est au $\mathrm{IV}^{\mathrm{e}}$ millénaire avant notre ère que les peuples blancs, Sémites, puis Indo-Européens - je dis «Albo-Européens » descendirent de l'extrême Nord jusque dans le bassin méditerranéen et à l'Est jusqu'à la mer d'Oman et à l'océan Indien [...] Je sais que certains auteurs, ne pouvant se consoler que les Égyptiens fussent des nègres, ont attribué l'invention de la première écriture aux Sumériens. C'est simplement remplacer «bonnet noir» par «noir bonnet». (1988 : 202-204)

En se faisant historien diachroniste, Senghor se veut surtout pédagogue humaniste : convaincre que la conviction hégélienne selon laquelle « l'Afrique n'a pas d'histoire » (1879) est une ruse de discours doublée d'une déformation historique, mais qui a fait fortune auprès d'une lignée d'intellectuels et d'hommes politiques français allant de Jules Ferry à Nicolas Sarkozy, en passant par Ernest Renan et Léon Blum ${ }^{36}$.

\footnotetext{
${ }^{36}$ On peut remarquer une forme de continuité sémantique et idéologique entre le «nègre inférieur » de Hegel (1822), le «droit des races supérieures » de Jules Ferry (juillet 1885), l'« homme africain qui n'est jamais entré dans l'histoire» de Nicolas Sarkozy (Juillet 2007 à Dakar) et la théorie des « civilisations qui ne se valent pas » de Claude Guéant (février 2012). Le trouble est encore plus grand si l'on se rappelle du discours d'Ernest Renan : « La conquête d'un pays de race inférieure, par une race supérieure, qui s'y établit pour le gouverner, n'a rien de choquant... Autant les conquêtes entre races égales doivent être blâmées, autant la régénération des races inférieures par les races supérieures est dans l'ordre providentiel de l'humanité $[\ldots] \mathrm{La}$ nature a fait une race d'ouvriers ; c'est la race chinoise, d'une dextérité de main merveilleuse sans presque aucun sentiment de l'honneur; une race de travailleurs de la terre, c'est le nègre ; une race de maîtres et de soldats, c'est la race européenne » (1871:13).
} 


\section{Senghor et les humanités africaines}

L'Afrique n'a pas seulement participé à l'histoire du monde et singulièrement à l'histoire contemporaine de la France - avec l'implication décisive des «Tirailleurs sénégalais » dans toutes ses guerres de libération -, mais des modèles d'organisation sociale, économique et politique ont toujours existé en Afrique précoloniale. Ces modèles - avec toutes les imperfections qui vont avec - sont porteurs de valeurs humaines et collectives, de visions solidaires du monde, de pédagogies écologiques et de procédures de justice et d'équité. Ces modèles d'organisation sociale anciens, Senghor les a longtemps étudiés dans de nombreux pays africains et voici comment il présente celui de sa propre ethnie :

Voici précisément les trois principales vertus qui font l'honnêtehomme dans ma culture. Ce sont le kersa, le teggin et le muñ. Le kersa, que l'on traduit souvent par « discrétion » ou «pudeur », est essentiellement la «maîtrise de soi ». C'est ce qui caractérise aussi bien le jeune homme ou la jeune fille modèles que la grande dame ou le sage. Le teggin est à rapprocher du verbe teg, « poser », « placer», «tenir », « conduire », c'est la manière de se tenir et de se conduire. Ce sont les bonnes manières. Je songe particulièrement à la politesse que l'on rend à chaque personne de son état, son rang et son mérite. Cette politesse est désignée par le mot teranga qui se rattache au verbe teral, «honorer» [...] Quant au muñ , c'est cette «patience» qui est aussi, plus qu'on ne le croit, une vertu africaine. Celle que l'on prête habituellement à notre classe laborieuse d'hommes libres : aux paysans, aux pasteurs et aux pêcheurs. (1988:14)

De son côté, Léon Blum déclarait dans la même Chambre des députés en 1925 : « Nous admettons le droit et même le devoir des races supérieures d'attirer à elles celles qui ne sont pas parvenues au même degré de culture ». 
L'explication de Senghor est ici d'abord sociolinguistique. Au-delà de la référence nationale, elle montre comment le substantif dialectal encode un univers de sens qui définit une forme de socialité africaine consacrée par des valeurs sociales (politesse, conduite, hospitalité, patience, etc.). La composition de ces mots n'indique ni une «race inférieure» ni une «population malheureuse » comme le déclare une tradition hégémoniste du discours occidental, mais au contraire, elle laisse voir de savants procédés morphologiques et dérivationnels du mot pour dire une façon magnifiée d'être et de vivre en société. Entre teg (poser) et teggin (respect), la continuité sémantique se réalise à partir d'une transition phonématique en /g/ doublé (ordre morphologique), mais cette continuité ne supprime pas l'opposition catégorielle entre le verbe et le substantif (ordre grammatical). La conceptualisation de l'éthique sociale dans la culture de Senghor signale donc d'abord une construction linguistique complexe qui aboutit à une sémantique du discours. Celle-ci peut même étendre graduellement les niveaux de sens, comme c'est le cas de la dérivation qualitative qui relie teral (accueillir) à teranga (hospitalité). Senghor donne sens à l'idée selon laquelle une langue secrète, encourage et véhicule à la fois une culture, et dans le cadre des langues africaines, c'est en considérant ensemble leurs procédés morphologiques, leurs structures syntaxiques, leurs variations phonologiques et leurs ressorts sémantiques qu'on peut valablement en percevoir les potentialités signifiantes et les ancrages culturels.

En remontant à la préhistoire des peuples du monde, en déconstruisant le discours hégémoniste occidental et en procédant à une linguistique des valeurs sociales africaines, Senghor élabore une approche minutieuse du fait social francophone. Cette approche sert un objectif personnel favorisé chez lui par ce que Pierre 
Bourdieu appelle une « trajectoire croisée » $(1991)^{37}$ qui permet à une personne d'occuper des positions dominantes dans des champs institutionnels différents d'une société donnée et à une période déterminée ${ }^{38}$. En effet, dès la fin de la Seconde Guerre mondiale, Senghor devient la figure centrale du champ politique sénégalais, d'abord comme Député du Sénégal à l'Assemblée nationale française (1945-1959), puis comme Président de la République (1960-1980). Il est aussi en même temps une figure centrale des lettres sénégalaises, africaines et francophones dès la parution de son premier recueil poétique Chants d'ombre en 1945.

Cette double présence privilégiée dans les champs politique et littéraire conforte une «trajectoire croisée » tout aussi privilégiée qui lui permet d'inspirer, d'intervenir, d'orienter et d'agir dans les deux champs institutionnels et bien audelà. C'est précisément à ce niveau que prend forme une subjectivité senghorienne performative qui le porte à traduire son savoir historique et conceptuel en visions et en moyens d'action sociopolitique. En tant que Président de la République, Senghor décide, en effet, de la nature du régime politique et socioéconomique à instaurer au Sénégal. Il supervise la construction d'un État démocratique basé sur un «multipartisme limité » et un « présidentialisme fort », il redessine totalement la carte administrative du pays, redéfinit les curricula scolaires et universitaires, centralise les politiques agricoles autour de la production de l'arachide et supervise la stratégie communicationnelle de l'État envers les ethnies et les confessions religieuses. Sur le plan culturel, et grâce

\footnotetext{
${ }^{37} \mathrm{La}$ « trajectoire croisée » est celle qui « conduit des positions dominantes dans le champ du pouvoir au champ de production culturelle, qui y occupe une position dominée, et, plus précisément aux positions temporellement dominantes dans le champ de production culturelle » (Bourdieu, 1991 : 10).
} 
toujours à sa posture présidentielle, Senghor a aussi les pleins pouvoirs pour bâtir une toute nouvelle infrastructure culturelle au Sénégal : il finance la construction du Théâtre national Daniel Sorano de Dakar où se tint le premier Festival mondial des arts nègres en avril $1966^{39}$, il finance aussi les constructions du Musée dynamique consacré à l'art traditionnel africain et du Village artisanal de Soumbédioune. Il ordonne l'établissement d'un réseau de salles de cinéma dans la capitale, soutient la mise sur pied de l'École picturale de Dakar et de l'École nationale des Beaux-Arts, inaugure la Manufacture des arts décoratifs de Thiès et fonde, entre autres, Les Nouvelles Éditions Africaines qui redynamisent la diffusion du livre au Sénégal et en Afrique de l'Ouest.

L'expression d'une subjectivité senghorienne performative ne se réduit donc pas à un simple onirisme poétique, mais matérialise un véritable projet national et africain pensé et exécuté à travers des politiques artistiques, administratives et sociales. Pour ce faire, il lui a fallu arrimer des habiletés construites (abilities) tout au long du $\mathrm{XX}^{\mathrm{e}}$ siècle à un capital agentif élevé (agency) soutenu par une « trajectoire croisée » rare chez les auteurs africains et francophones.

Fort de son savoir doublement intellectuel et littéraire, précolonial et colonial, culturel et sociolinguistique, et servi par une «trajectoire croisée » favorable, Senghor veut démontrer par le verbe et par l'action que même consécutif au rapport colonial avec la France, le fait francophone observable à l'échelle de

\footnotetext{
${ }^{39}$ À ce Festival ont participé des personnalités culturelles majeures de l'époque comme Aimé Césaire, Duke Elligton, Jean Price-Mars, Aminata Fall, Wolé Soyinka, Joséphine Baker, Langston Hughes ou André Malraux. Revenant sur ce premier événement culturel d'envergure sur le continent, É. Ficquet et L. Gallimardet notent que « d'importants moyens budgétaires et humains furent investis dans ce projet, conçu par le chef de l'État comme une priorité nationale. Les plus hautes instances du pouvoir sénégalais furent mobilisées sous la supervision directe de la présidence de la République, relayée par un comité interministériel chargé de secteurs logistiques spécifiques (transports, logements, communication, etc.)» (2009 : 10).
} 
chaque pays n'est pas une donnée figée mais plutôt un devenir collectif à construire. Il n'est pas une histoire subie mais un présent à inventer et un pari sur l'avenir. Il n'est pas une distribution verticale des rôles mais une participation horizontale à l'œuvre humaine enracinée en tous lieux et en toutes civilisations. Des Antilles à l'Afrique subsaharienne, du Maghreb aux pays de l'océan Indien, du Québec au Moyen Orient, il faut donner à ce fait francophone une couleur locale, il faut en faire ressortir toutes les expressions artistiques, lui faire dire des humanités partagées dans des langages puissamment hétéroclites. Bref, il faut lui faire inventer toutes les possibilités d'un nouveau « vivre-ensemble » au sens où l'entend précisément Alain Touraine ${ }^{40}$.

$\mathrm{Au}$ terme de cette réflexion sur la réalité du fait francophone tel que le conçoit Senghor, il semble que l'auteur de La poésie de l'action (1980) y voit d'abord une communauté plurielle qui assume son passé pour difficile soit-il et qui vit et accepte sa disparité sociolinguistique et créative. La meilleure façon d'assumer cette disparité fondatrice est de laisser libre cours à l'énonciation subjective qui remet « l'Homme dans le système », comme le suggère Senghor lui-même, c'està-dire au centre des enjeux relationnels et civilisationnels du monde :

L'existence se fonde sur l'existence pour s'épanouir en existence. D'où la place privilégiée qu'occupe l'Homme dans le système, en sa qualité d'existant actif, capable de renforcer sa force, de se réaliser en personne, c'est-à-dire en existant de plus en plus libre au sein d'une communauté solidaire. (1969:8)

\footnotetext{
${ }^{40}$ Le « vivre-ensemble » est un projet collectif qui «combine l'unité d'une société avec la diversité des personnalités et des cultures en plaçant l'idée de Sujet personnel au centre de la réflexion et de l'action » $(1997: 18)$. Le « vivre-ensemble » se réalise autour de principes clefs comme « intérêt général », « service public », « expression des minorités », « reconnaissance des droits », « citoyenneté partagée », « laïcité », etc.
} 
Il faut croire que ce projet de toujours remettre «l'Homme dans le système » reste le défi quotidien du fait francophone contemporain qui est nécessairement multiforme et dynamique. Il est également celui qui rend Senghor puissamment «moderne » car c'est sous ce même rapport que l'épidémie du Coronavirus de 2020-2021 démontre jusqu'à quel point cet «Homme » menacé est le même sur tous les points du Globe. C'est sous ce même rapport aussi que se repensent les pratiques littéraires francophones de nos jours, celles qui accordent de plus en plus d'importance aux pragmatiques textuelles autant qu'aux filiations épitextuelles, aux hybridités auctoriales autant qu'aux énonciations postcoloniales. La modernité de Senghor reste alors performative car elle réfère à toutes les formes de subjectivités qui figurent un monde... pour mieux dire le monde. 


\section{Bibliographie}

Bourdieu, Pierre, Les règles de l'art. Genèse et structure du champ littéraire, Paris, Seuil, 1992.

Bourdieu, Pierre, «Le champ littéraire », Actes de la recherche en sciences sociales, 89, 1991, pp. 3-46.

Clavaron, Yves, «Villes et espaces africains : pour une géocritique en contexte postcolonial », Présence francophone, n $^{\circ} 88,2017$, pp.12-24.

Diouf, Mbaye, «La philosophie senghorienne du dialogue interreligieux », dans Pierre Halen et Florence Paravy (dir.), Littératures africaines et spiritualité, Bordeaux, Presses Universitaires de Bordeaux, coll. «Littératures des Afriques », 2, 2016, pp.181-198.

Diouf, Mbaye, (dir.), «Les figurations spatiales francophones: essais géocritiques », Présence Francophone, n88, automne 2017.

Ferry, Jules, « Les fondements de la politique coloniale », Discours, 28 juillet $1885 . \quad$ [http://www2.assemblee-nationale.fr/decouvrir-lassemblee/histoire/grands-moments-d-eloquence/jules-ferry-28-juillet-1885].

Ficquet, Éloi ; Gallimardet, Lorraine, « Enjeux du colloque et de l'exposition du Premier Festival mondial des arts nègres de Dakar en 1966 », Revue Gradhiva, 10, 2009, pp.134-155.

Hegel, G. W. Friedrich, La raison dans l'histoire, Paris, Éditions 10/18, 2003 [1822-1830].

Hugo, Victor, Discours sur l'Afrique, 18 mai 1879. [http://dormirajamais.org/hugo/].

Hume, David, Traité de la nature humaine, Paris, Flammarion, 1999 [1739].

Montesquieu, De l'esprit des lois, Paris, Flammarion, 1993 [1748].

Moura, Jean-Marc, Littératures francophones et théorie postcoloniale, Paris, PUF, 2013 [1999]. 
Renan, Ernest, Réforme intellectuelle et morale, Paris, Calmann Lévy, 1871.

Sanogo, Amidou, «Subjectivité et représentation socio-discursive dans la littérature négro-africaine d'expression française ", Recherches Francophones, $\mathrm{n}^{\circ} 1,2021$.

Semprun, Jorge, L'écriture et la vie, Paris, Gallimard, 1994.

Semujanga, Josias, Le génocide, sujet de fiction? Québec, Nota Bene, 2008.

Senghor, Léopold Sédar, Euvre poétique, Paris, Seuil, 1990.

Senghor, Léopold Sédar, Ce que je crois, Paris, Grasset, 1988.

Senghor, Léopold Sédar, Discours de remerciement et de réception à l'Académie française, Paris, Seuil, 1984.

Senghor, Léopold Sédar, La poésie de l'action, conversations avec Mohamed Aziza, Paris, Stock, 1980.

Senghor, Léopold Sédar, Négritude, arabité, francité, Beyrouth, Dar Al-Kitab Allubnani, 1969.

Taylor, Charles, Multiculturalisme: différence et démocratie, Paris, Flammarion, 2009 [1994].

Touraine, Alain, Pourrons-nous vivre ensemble ? Paris, Fayard, 1997.

Voltaire, Essai sur les mours et l'Esprit des Nations, Oxford, Voltaire Foundation, 2009 [1756]. 